\title{
GETTING TO KNOW THE NEAREST STARS: INTERMITTENT RADIO EMISSION FROM ROSS 614
}

\author{
M. Knapp*, D. Winterhalter ${ }^{\dagger}$, and T. Bastian ${ }^{\ddagger}$
}

\begin{abstract}
We present results from a VLA survey of the 10 nearest stars in the Northern sky in $\mathrm{P}, \mathrm{L}$, and $\mathrm{S}$ bands. The purpose of this survey is to set firm upper limits on radio emission from stars and yet-undiscovered planets orbiting our nearest stellar neighbors. Bounding the radio emission from these stars will inform discussions of habitability for any planets discovered in these systems. We report strong detections of stellar radio emission at $3 \mathrm{GHz}$ from Ross 614 during March 2016, but not in later observations. We also present upper limits on radio emission from the other survey targets.
\end{abstract}

\footnotetext{
* Department of Earth, Atmospheric, and Planetary Science, Massachusetts Institute of Technology (MIT), Cambridge, MA, USA

${ }^{\dagger}$ NASA Jet Propulsion Laboratory, Caltech, Pasadena, CA, USA

$¥$ National Radio Astronomy Observatory (NRAO), Charlottesville, VA, USA
} 
